\title{
STRONGLY DAMPED WAVE EQUATION AND ITS YOSIDA APPROXIMATIONS
}

\author{
Matheus C. Bortolan - Alexandre N. Carvalho
}

\begin{abstract}
In this work we study the continuity for the family of global attractors of the equations $u_{t t}-\Delta u-\Delta u_{t}-\varepsilon \Delta u_{t t}=f(u)$ at $\varepsilon=0$ when $\Omega$ is a bounded smooth domain of $\mathbb{R}^{n}$, with $n \geq 3$, and the nonlinearity $f$ satisfies a subcritical growth condition. Also, we obtain an uniform bound for the fractal dimension of these global attractors.
\end{abstract}

\section{Introduction}

We study the continuity of global attractors of the following semilinear evolution equation of second order in time

$$
\left\{\begin{array}{l}
u_{t t}-\Delta u-\Delta u_{t}-\varepsilon \Delta u_{t t}=f(u), \quad t>0, \\
\left(u(0), u_{t}(0)\right)=\left(u_{0}, v_{0}\right), \\
\left.u\right|_{\partial \Omega}=0,
\end{array}\right.
$$

and we give an uniform bound for the fractal dimension of these global attractors.

We know that, for $\varepsilon=0$, this equation is the usual strongly damped wave equation, and its asymptotic dynamics - related to global atrtactors - has already been vastly explored; see for instance [6], [7], [9], [12], [15], [22], [23], [26]-[28].

2010 Mathematics Subject Classification. 34D45, 37L30.

Key words and phrases. Global attractor, Yosida approximation, continuity of attractors, fractal dimension.

The first named author partially supported by FAPESP 2012/23724-1.

The second named author partially supported by CNPq 305230/2011-5. 
However, for each $\varepsilon>0$ fixed, we have a special form of the improved Boussinesq equation (see [4], [19], [20], [25]) with damping $-\Delta u_{t}$, which, among other things, is used to describe ion-sound waves in plasma (see [20], [21]).

For each $\varepsilon>0$ fixed, this equation has been studied in [8], in terms of existence and uniqueness of solutions, existence of global attractors and asymptotic bootstrapping; in this case, the linear part of the equation (after a change of variables) is a bounded operator. Here, since we want to study the continuity of attractors at $\varepsilon=0$, we will use the properties of the limiting problem with $\varepsilon=0$ (local and global well posedness, regularity and existence of global attractors) as reported in [6], [7].

Throughout this paper, we will assume that $f: \mathbb{R} \rightarrow \mathbb{R}$ is a continuously differentiable function, respecting a growth condition with subcritical exponent; that is, there exist constants $c>0$ and $\rho<(n+2) /(n-2)$ such that for all $s_{1}, s_{2} \in \mathbb{R}$

$$
\left|f\left(s_{1}\right)-f\left(s_{2}\right)\right| \leq c\left|s_{1}-s_{2}\right|\left(1+\left|s_{1}\right|^{\rho-1}+\left|s_{2}\right|^{\rho-1}\right),
$$

and also, if $\lambda_{1}$ denotes the first eigenvalue of $-\Delta$ with Dirichlet boundary conditions in $\Omega$, we assume the following dissipation condition

$$
\limsup _{|s| \rightarrow \infty} \frac{f(s)}{s}<\lambda_{1} .
$$

To begin our study, we will write further $A$ for $-\Delta$ with the Dirichlet boundary conditions. Our problem then takes the form

$$
\left\{\begin{array}{l}
u_{t t}+A u+A u_{t}+\varepsilon A u_{t t}=f(u), \quad t>0, \\
(u(0), v(0))=\left(u_{0}, v_{0}\right) .
\end{array}\right.
$$

and it is well-known that $A: H_{0}^{1}(\Omega) \cap H^{2}(\Omega) \subset L^{2}(\Omega) \rightarrow L^{2}(\Omega)$ is a closed, densely define operator which has the following properties:

(O1) $A$ is self-adjoint with compact resolvent;

(O2) $A$ is an operator of positive type;

(O3) $\sigma(A)=\sigma_{p}(A)=\left\{\lambda_{n}\right\}_{n \in \mathbb{N}}, \lambda_{1}>0, \lambda_{i} \leq \lambda_{i+1}$, for all $i \geq 1$ (repeated to take into account the multiplicity), $\lambda_{n} \stackrel{n \rightarrow \infty}{\longrightarrow} \infty$ and if $v_{n} \in L^{2}(\Omega)$ are unitary eigenvectors associated with $\lambda_{n}$ then $\left\{v_{n}\right\}_{n \in \mathbb{N}}$ constitutes an orthonormal basis for $L^{2}(\Omega)$.

Remark 1.1. We included in Appendix A the proof of the main results of functional analysis we will use, in order to make explicit the uniformity of the constants obtained for $\varepsilon \in[0,1]$.

The key point in our analysis is the observation that the differential equation in (1.4), for $\varepsilon>0$, can be obtained from its limit, for $\varepsilon=0$, with a suitable exchange of the unbounded operator $A$ by its Yosida approximation $\Lambda_{\varepsilon}$ (see 